\title{
MOLECULAR DOCKING STUDIES OF CURCUMA LONGA AND ALOE VERA FOR THEIR POTENTIAL ANTICANCER EFFECTS
}

\author{
PARUL TRIPATHI, SAAD SABIR SIDDIQUI, ANJU SHARMA, PARUL JOHRI, ADITI SINGH*
}

Amity Institute of Biotechnology, Amity University Uttar Pradesh, Lucknow Campus, Malhaur, Gomti Nagar Extension, Lucknow - 226 028, Uttar Pradesh, India. Email: asingh3@lko.amity.edu

Received: 28 November 2017, Revised and Accepted: 12 January 2018

ABSTRACT

Objective: In this paper, docking study is presented to use these phytocompounds for their prospective role in various types of cancers.

Methods: A group of the different set of phytocompounds (aloesin, barbaloin, curcumin, and emodin) were taken and docked into the active sites of Topoisomerase I, a 91-kDa monomer (having 765 amino acids), is encoded by a single copy gene (Top 1) located on chromosome 20q12-13.2 using Autodock4 Software. The docking studies of the selected proteins were also docked to study the anticancerous property of the selected phytocompounds.

Result: These studies were based on binding energy, docking energy and other relevant scores that revealed emodin could be the potential lead molecule for the inhibition of signal potent for different types of cancer. Furthermore, the important residues for potential drug target were identified.

Conclusion: This paper is an initial step toward a rational design of novel selective and potent phytocompounds inhibitors for the treatment of deadly disease cancer.

Keywords: Anticancer, Docking, Phytocompounds.

(C) 2018 The Authors. Published by Innovare Academic Sciences Pvt Ltd. This is an open access article under the CC BY license (http://creativecommons. org/licenses/by/4. 0/) DOI: http://dx.doi.org/10.22159/ajpcr.2018.v11i4.23995

\section{INTRODUCTION}

Plants and their extracts have been used to treat human diseases since ancient time. Among the many medicinal and therapeutic properties, plants such as Andrographis paniculata, Arachis hypogaea, Datura inoxia, Aloe vera, and Curcuma longa have also been explored for their cancer chemotherapeutic potential [1-5]. In C. longa (Indian spice turmeric) the most active component is curcumin, the yellow substance belonging to polyphenols superfamily. It has been extensively used in Ayurvedic medicine for centuries, as it is nontoxic and has a variety of therapeutic properties including antioxidant, analgesic, anti-inflammatory, and antiseptic activity [6,7]. Recently, curcumin has also been found to possess anticancer activities because of its effect on a variety of biological pathways involved in mutagenesis, oncogene expression, cell cycle regulation, apoptosis, tumorigenesis, and metastasis [7-9].

The herb A. vera is widely used in Ayurveda, homoeopathy as well as allopathy for its wide medicinal properties. The most active plant part extract is leaf gel which has more than 200 different biologically active substances having antimicrobial, anti-inflammatory, anthelmintic, wound healing, and also anticancer activities [10-12]. The most significant anthraquinones in aloe gel are aloesin, aloe-emodin $(\mathrm{AE})$, and barbaloin. Aloesin is a potent and selective inhibitor of tyrosinase and exhibits direct inhibitory effects on melanogenesis [13]. However, little is known about the role of aloesin in anticancer activity. Barbaloin is C-glucoside of $\mathrm{AE}$ anthrone, and has strong inhibitory effect on histamine release, cathartic, and anticancer activity. AE, the hydroxyanthraquinone has been studied to possess in vitro and in vivo anti-neuroectodermal tumor activity [14].

Many cell cycle molecules and genes have been studied to be involved in cancer pathogenesis. Targeting such proteins and molecules may be a good strategy for anticancer therapy. In the current study, molecular docking was performed to find out the target protein for these four potential anticancer compounds (aloesin, barbaloin, curcumin, and emodin), their respective binding energies along with the number of hydrogen bonds and other hydrophobic interactions.

\section{METHODS}

\section{Structure retrieval}

Selection of the target proteins has been done through literature search [15-18]. Proteins who have anticancer properties been selected for the study. The three-dimensional crystal structures of all 14 target proteins were retrieved from the protein data bank (PDB) (https:// www.rcsb.org/pdb/home/home.do) (Table 1).

C. longa and A. vera are very well known for their therapeutic properties, so it was decided to check their anticancerous effect in silico. The two-dimensional structures of ligand molecules (aloesin, barbaloin, curcumin, and emodin) were downloaded from PubChem database (https://pubchem.ncbi.nlm.nih.gov) (Table 2).

\section{Docking studies}

The docking studies were performed by AutoDock 4 Software. AutoDock 4 Software serves as a valid and acceptable docking application to study the interactions of small compounds with proteins. All protein structures and ligands were prepared for docking studies using various parameters such as addition of polar hydrogen atoms, merging of non-polar hydrogen atoms and defining the rotatable bonds for each ligand. Finally, Kollman united atom charge, and atom type parameter was added. The grid parameters $\left(60^{*} 60^{*} 60\right)$ for all proteins were set in such a way that it includes the active site. The 
Lamarckian genetic search algorithm was employed, and docking was set to 20 runs. All other parameters were set to the default values such as maximum number of energy evaluation was set as 25,00,000 per run and maximum number of generations in the genetic algorithm was increased to $2,70,000$ (Fig. 1).

\section{RESULTS}

The results obtained after docking aloesin, barbaloin, curcumin, and emodin (Table 2) with 14 different proteins (Table 1) were evaluated on the basis of energy. The binding energy obtained from docking interactions, ligand efficiency and inhibition constant values calculated by AutoDock 4 were considered as the primary parameters for studying the docking behavior of ligands with our proteins (Table 3). Binding energy is a measure of the affinity of ligand-protein complex or is the difference between the energy of complex and the sum of energies of each molecule separately. Inhibition constant is an indication of how potent an inhibitor is, it is concentration required to produce half maximum inhibition. The ligand efficiency is binding energy per atom of ligand to protein and is defined as the calculated pKi divided by the number of heavy atoms in the ligand; the smaller is the ligand efficiency, the more promising is the docking. Optimal parameters were used as criteria to interpret the best pose among the 10 conformations generated by the software AutoDock 4. The molecular docking studies provide valuable information about the residues involved in the enzyme-substrate interaction.

Table 1: Proteins used as target for docking studies

\begin{tabular}{lllll}
\hline S. No & Name & PDB ID & Length & Experimental info \\
\hline 1 & Topo I & $1 \mathrm{k} 4 \mathrm{t}$ & 592 & X-ray diffraction \\
2 & NF-KB & $1 \mathrm{nfk}$ & 312 & X-ray diffraction \\
3 & Caspase 3 & $1 \mathrm{pau}$ & 504 & X-ray diffraction \\
4 & Caspase 8 & $1 \mathrm{qtn}$ & X-ray diffraction \\
5 & PKC & $1 \mathrm{yrk}$ & X-ray diffraction \\
6 & CDK2 & $2 \mathrm{bhh}$ & 13 & X-ray diffraction \\
7 & EGFR & $2 \mathrm{itx}$ & 298 & X-ray diffraction \\
8 & MMP-9 & $2 \mathrm{ovx}$ & 324 & X-ray diffraction \\
9 & CFLIP & $3 \mathrm{~h} 13$ & 159 & X-ray diffraction \\
10 & COX-2 & $3 \ln 1$ & 271 & X-ray diffraction \\
11 & mTOR & $3 \mathrm{oaw}$ & 552 & X-ray diffraction \\
12 & HER2 Protein & $3 \mathrm{pp} 0$ & 951 & X-ray diffraction \\
13 & Topo II $\beta$ & $3 \mathrm{q} \times 3$ & 308 & X-ray diffraction \\
14 & MPO & $4 \mathrm{c} 1 \mathrm{~m}$ & 12.98 & $2.2 \AA$ \\
\hline
\end{tabular}

PDB: Protein data bank, EGFR: Epidermal growth factor receptor

Table 2: Ligand molecules used for docking studies

\begin{tabular}{|c|c|c|c|c|c|}
\hline S. No & Name & PubChem CID & Chemical formula & Molecular weight $(\mathrm{g} / \mathrm{mol})$ & 2D structure \\
\hline 1 & Aloesin & 160190 & $\mathrm{C}_{19} \mathrm{H}_{22} \mathrm{O}_{9}$ & $394.376 \mathrm{~g} / \mathrm{mol}$ & \\
\hline 2 & Barbaloin & 12305761 & $\mathrm{C}_{21} \mathrm{H}_{22} \mathrm{O}_{9}$ & $418.398 \mathrm{~g} / \mathrm{mol}$ & \\
\hline 3 & Curcumin & 969516 & $\mathrm{IC}_{21} \mathrm{H}_{20} \mathrm{O}_{6} / \mathrm{C}_{21} \mathrm{H}_{20} \mathrm{O}_{6}$ & $368.385 \mathrm{~g} / \mathrm{mol}$ & \\
\hline 4 & Emodin & 3220 & $\mathrm{C}_{15} \mathrm{H}_{10} \mathrm{O}_{5}$ & $\begin{array}{l}270.24 \\
\mathrm{~g} / \mathrm{mol}\end{array}$ & \\
\hline
\end{tabular}


Aloesin showed the lowest binding energy of $-3.16 \mathrm{kcal} / \mathrm{mol}$, ligand efficiency of -0.11 , and inhibition constant of $4.79 \mu \mathrm{M}$, with Protein kinase C (1yrk) out of the 14 proteins. The best-docked conformation of aloesin showed hydrogen-binding interactions with the active residues Lys96, Leu3, and Leu122 of 1yrk with a binding free energy $-3.16 \mathrm{kcal} / \mathrm{mol}$ (Fig. 2).

Barbaloin showed the lowest binding energy of $-2.9 \mathrm{kcal} / \mathrm{mol}$ and $-2.93 \mathrm{kcal} / \mathrm{mol}$ with protein $1 \mathrm{qtn}$ and 3oaw, respectively, out of 14 proteins selected as a receptor for docking. Both proteins were found to have same ligand efficiency $(-0.1)$ and inhibition constant $(7.16 \mu \mathrm{M})$ with barbaloin inferring equal affinity of binding. The best-docked conformation of barbaloin showed hydrogen-binding interactions with the active residues Asn452, Ser451, Tyr412, Thr503, Gly368, and Arg413 of 1qtn (Fig. 3).

When barbaloin was docked with protein 3oaw, hydrogen bonds are formed with Asn452, Ser451, Tyr412, Thr503, Gly368, and Arg413 residues (Fig. 4).

Curcumin binds best with epidermal growth factor receptor (2itx) getting the binding energy of $-4.0 \mathrm{kcal} / \mathrm{mol}$. The ligand efficiency and inhibition constant of curcumin with 2itx are 1.18 and $-0.15 \mu \mathrm{M}$ respectively. The best-docked conformation of curcumin showed hydrogen-binding interactions with the active residues Asn700, Pro999, Gln701, Glu697, and Pro1019 of 2itx (Fig. 5).

Emodin binds best with topo I $(1 \mathrm{k} 4 \mathrm{t})$ getting the binding energy of $-5.71 \mathrm{kcal} / \mathrm{mol}$. The measured ligand efficiency and inhibition constant with $1 \mathrm{k} 4 \mathrm{t}$ observed are 65.38 and $-0.29 \mu \mathrm{M}$, respectively. The bestdocked confirmation of emodin showed hydrogen-binding interactions with active residues Trp416, Glu356, and lle377 active residues of $1 \mathrm{k} 4 \mathrm{t}$ (Fig. 6).

The H-bonding stabilizes the ligand and the target site and helps alter binding affinity and drug efficacy. The result presented in this paper provides a novel in silico based approach to design drug lead at the hydrophobic core of ligand and protein interphase. The weak hydrogen bonds can be broken and exchanged for another kind of bond depending on the chemical environment at the target, ligand, and target-ligand interphase. The summarized details of the hydrogen bonds studied are listed in Table 4.

Table 3: Describes the data obtained from docking log file for every ligand-receptor docking

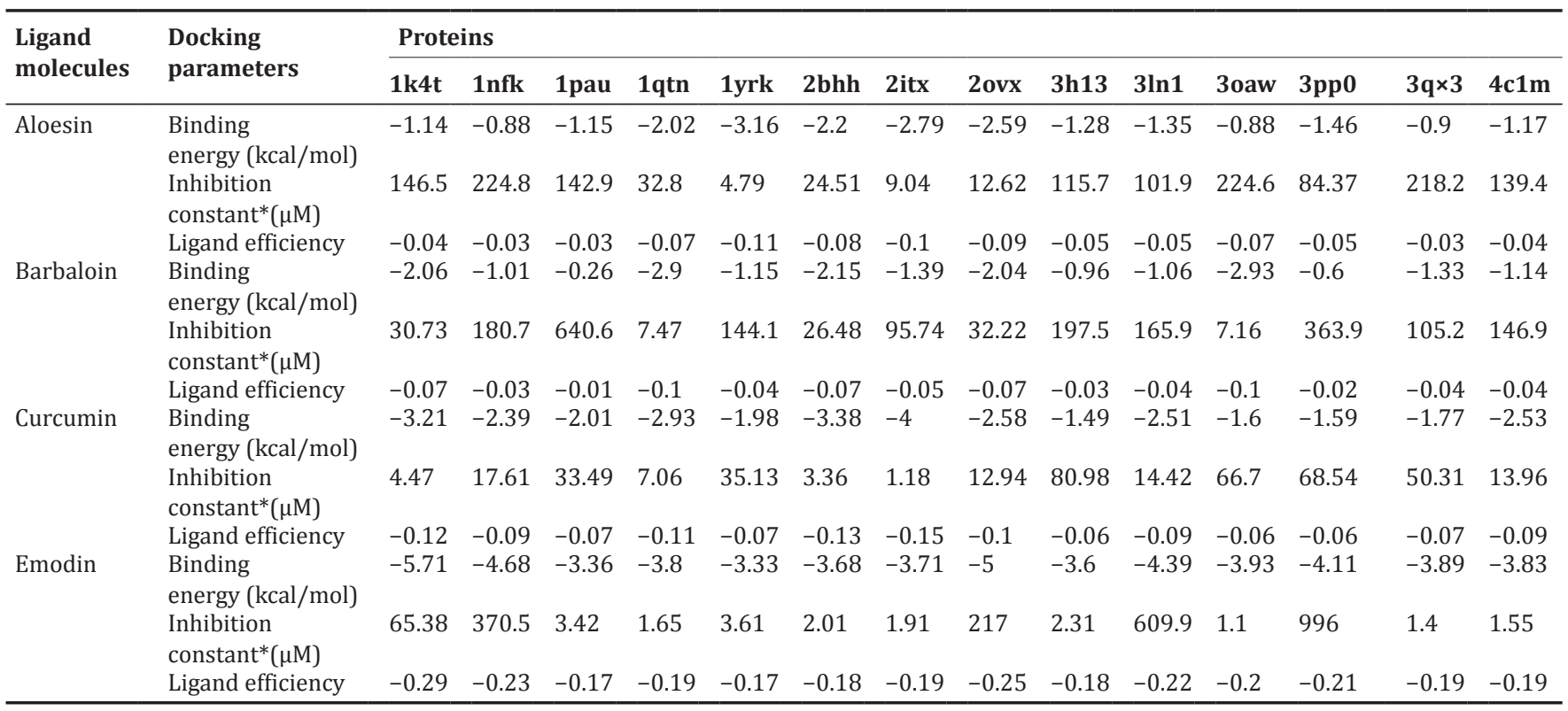

*The change in binding free energy is related to the inhibition constant using the equation: $\Delta \mathrm{G}=\mathrm{RT}$ in Ki, where $\mathrm{R}$ is the gas constant 1.987 cal $\mathrm{K}-1 \mathrm{~mol}-1$, and $\mathrm{T}$ is the absolute temperature assumed to be $298.15 \mathrm{~K}$

Table 4: Summarized hydrogen bonding interactions for all the proteins and their ligands

\begin{tabular}{lllll}
\hline S. No & Protein name & Compound & Interaction & Amino acid involved in interaction \\
\hline 1 & Aloesin & Protein kinase C (1yrk) & Hydrogen bond & Lys96, Leu3, Leu122 \\
2 & Barbaloin & Caspase 8 (1qtn) & Hydrogen bond & Asn452, Ser451, Tyr412, Thr503, Gly368, Arg413 \\
3 & Barbaloin & mTOR (3oaw) & Hydrogen bond & Asn452, Ser451, Tyr412, Thr503, Gly368, Arg413 \\
4 & Curcumin & EGFR (2itx) & Hydrogen bond & Asn700, Pro999, Gln701, Glu697, Pro1019 \\
5 & Emodin & Topo I (1k4t) & Hydrogen bond & Trp416, Glu356, lle377 \\
\hline
\end{tabular}

EGFR: Epidermal growth factor receptor

Table 5: Energy in KJ/mol for ligand complex with top scoring proteins

\begin{tabular}{|c|c|c|c|c|}
\hline S. No & Ligand (zinc IDs/name) & Protein (PDB ID) & VDW (kcal/mol) & Electrostatic energy $(\mathrm{KJ} / \mathrm{mol})$ \\
\hline 1 & Aloesin & $1 y r k$ & 6.82 & -0.1 \\
\hline \multirow[t]{2}{*}{2} & Barbaloin & a) 1 qtn & -8.53 & -0.64 \\
\hline & & b) 3oaw & -8.99 & -0.22 \\
\hline 3 & Curcumin & 2itx & -9.32 & -0.19 \\
\hline 4 & Emodin & $1 \mathrm{k} 4 \mathrm{t}$ & -8.44 & -0.09 \\
\hline
\end{tabular}

PDB: Protein data bank, VDW: Van der Waals 


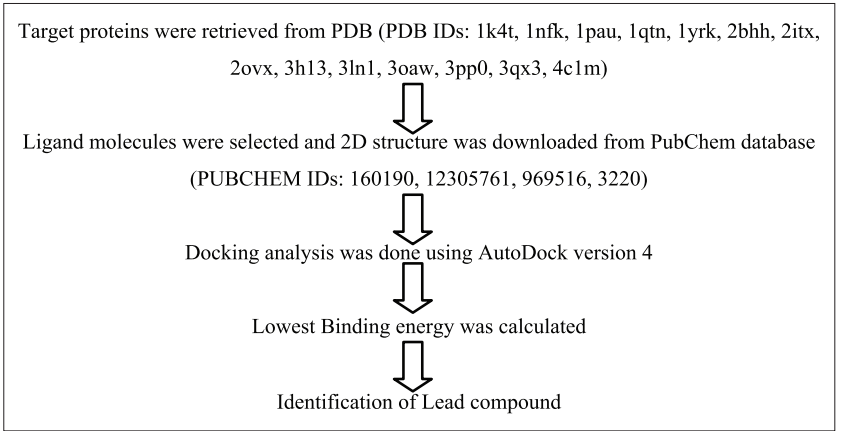

Fig. 1: Flowchart for methodology

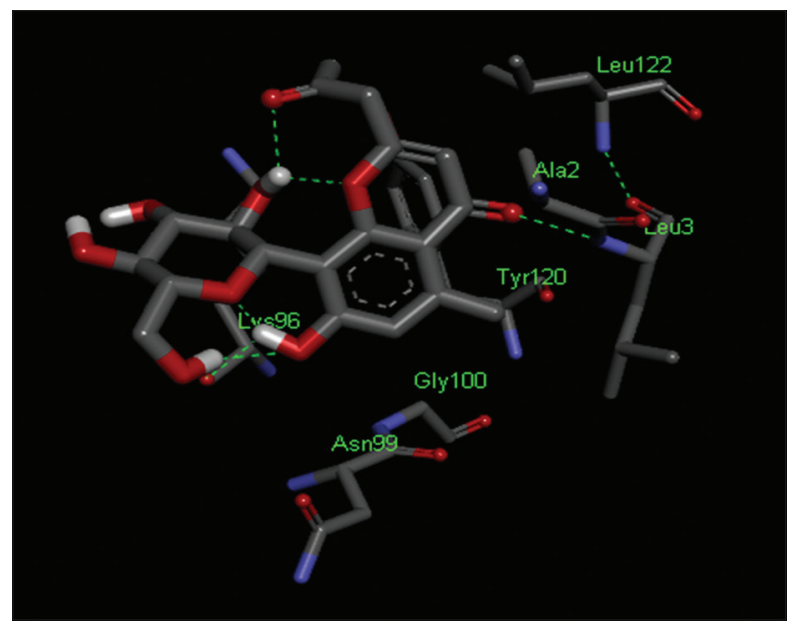

Fig. 2: Docked complex of aloesin (160190) with protein kinase $\mathrm{C}$ (1yrk) in ribbon representation and their interacting residues; dotted lines showing the hydrogen bond interaction

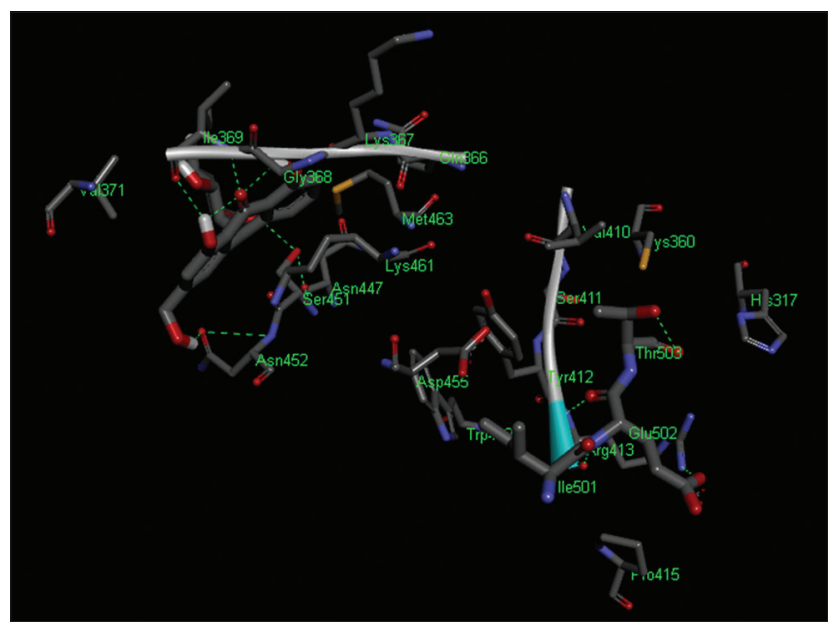

Fig. 3: Docked complex of barbaloin (12305761) with caspase 8 (1qtn) in ribbon representation and their interacting residues; dotted lines showing the hydrogen bond interaction

Ligands and best-docked proteins along with their van der Waals value and electrostatic energy (KJ/mol) are shown in Table 5.

\section{CONCLUSION}

The protein-ligand interaction plays a significant role in structurebased drug designing. The data suggest that emodin and curcumin have potential therapeutic value in cancer. Since the compounds do not exhibit side effects, it has been designated for several clinical trials as a treatment of human cancer, yet its mechanism of action remains

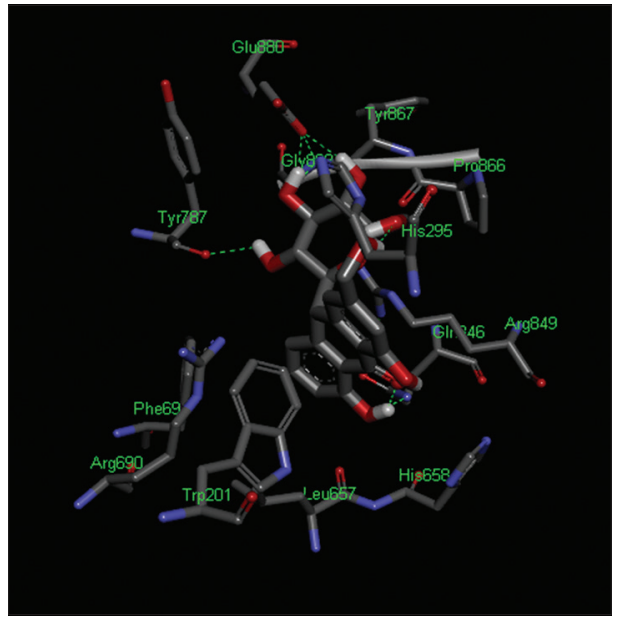

Fig. 4: Docked complex of barbaloin (12305761) with mTOR (3oaw) in ribbon representation and their interacting residues; dotted lines showing the hydrogen bond interaction

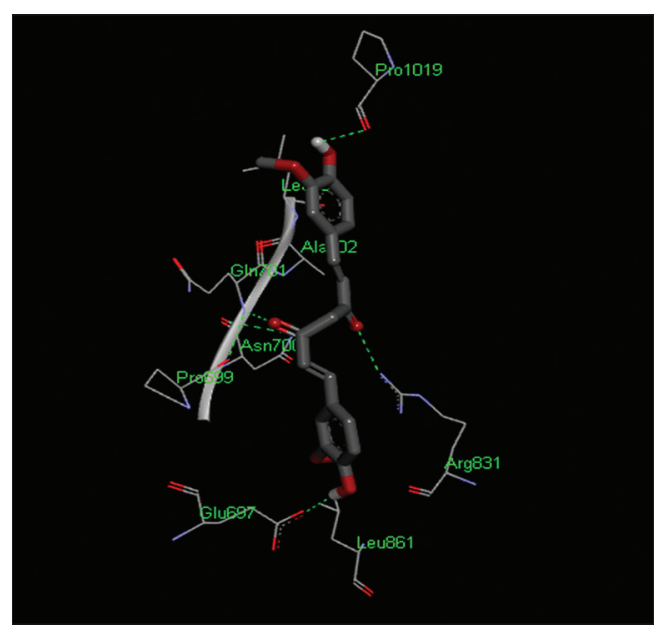

Fig. 5: Docked complex of curcumin (969516) with epidermal growth factor receptor (2itx) in ribbon representation and their interacting residues; dotted lines showing the hydrogen bond interaction

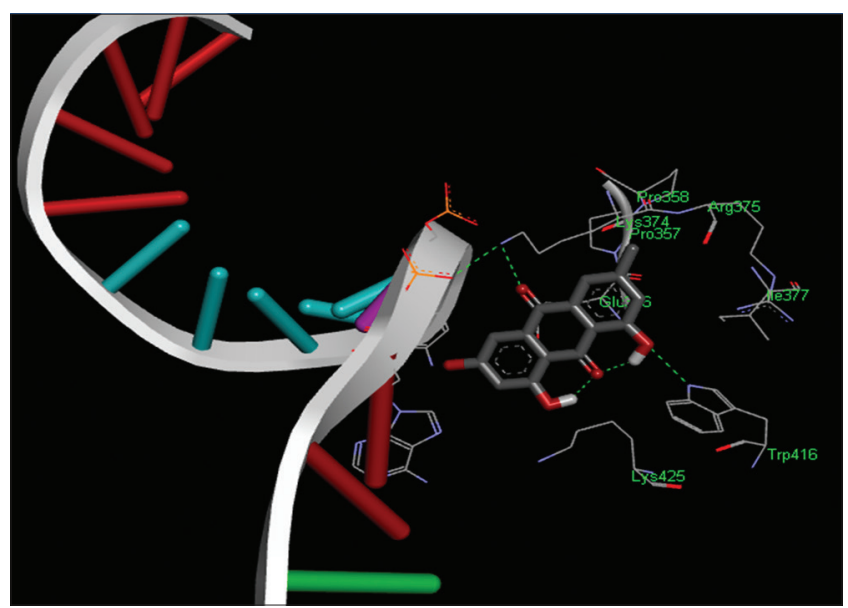

Fig. 6: Docked complex of emodin (3220) with topo I (1k4t) in ribbon representation and their interacting residues; dotted lines showing the hydrogen bond interaction

unknown. The ligand emodin showed the lowest binding affinity $(-5.71 \mathrm{kcal} / \mathrm{mol})$ against Topoisomerase I (PDB ID: $1 \mathrm{k} 4 \mathrm{t})$. Human 
deoxyribonucleic acid (DNA) Topoisomerase I catalyze single-strand breaking and rejoining in a DNA double helix in an ATP-independent manner [19].

Therefore, this study states the importance of compounds from $C$. longa and A. vera and their use to enhance protein-ligand studies, in silico. From the docking results, it is possible to conclude that emodin could be a potential Topo I inhibitor. In this study, the molecular docking was applied to explore the binding mechanism and to correlate its docking score with the activity of plant-derived compounds. The results of our present study can be useful for the design and development of novel compounds having better inhibitory activity against several types of cancers. This potential agent will be a promising candidate and can further be validated in wet lab studies for its proper function and can proceed for clinical trials [20].

\section{AUTHOR'S CONTRIBUTION}

Authors Sharma A, Tripathi $\mathrm{P}$ and Siddiqui SS make substantial contributions to conception and design, acquisition of data, lab work and analysis and interpretation of data. Authors Singh A and Johri P participate in drafting the article or revising it critically for important intellectual content. All the authors gave final approval of the version to be submitted and any revised version.

\section{CONFLICTS OF INTEREST}

The authors have declared that no conflict of interest exists.

\section{REFERENCES}

1. Huidrom S, Deka M. Determination of antioxidant property of Andrographis paniculata. Indian J Drugs Dis 2012;1:12-7.

2. Tripathi $P$, Singh A. Indigenous Asian plants against cancer: A comprehensive review. Int J Plant Res 2015;5:80-6.

3. Tripathi P, Singh A. Natural resources from plants in the treatment of cancer: An update. Asian J Pharm Clin Res 2017;10:13-22.

4. Vermillion K, Holguin FO, Berhow MA, Richins RD, Redhouse T, O'Connell MA, et al. Dinoxin B, a withanolide from Datura inoxia leaves with specific cytotoxic activities. J Nat Prod 2011;74:267-71

5. Yadav DN, Yogesh K, Aswani A. Antioxidant activity of peanut (Arachis hypogaea L.) skin extract: Application in soybean and mustard oil. Int J
Food Process Technol 2014;1:26-31

6. Akram M, Shahab-uddin, Ahmed A, Usmanghani K, Hannan A, Mohiuddin E, et al. Curcuma longa and curcumin: A review article. Rom J Biol Plant Biol 2010;55:65-70.

7. Wilken R, Veena MS, Wang MB, Srivatsan ES. Curcumin: A review of anti-cancer properties and therapeutic activity in head and neck squamous cell carcinoma. Mol Cancer 2011;10:12.

8. Vallianou NG, Evangelopoulos A, Schizas N, Kazazis C. Potential anticancer properties and mechanisms of action of curcumin. Anticancer Res 2015;35:645-51.

9. Jerah A, Hobani Y, Kumar BV, Bidwai A. Curcumin binds in silico to anti-cancer drug target enzyme MMP-3 (human stromelysin-1) with affinity comparable to two known inhibitors of the enzyme. Bioinformation 2015;11:387-92.

10. Sahu PK, Giri DD, Singh R, Pandey P, Gupta S, Shrivastava AK, et al. Therapeutic and medicinal uses of Aloe vera: A review. Pharmacol Pharm 2013;4:599-610.

11. Hashemi SA, Madani SA, Abediankenari S. The review on properties of Aloe vera in healing of cutaneous wounds. Biomed Res Int 2015;2015:714216.

12. Tripathi P, Singh A. Aloe vera: Plant with diverse therapeutic properties. Everyman's Sci 2016;1:6-9.

13. Zhang LQ, Lv RW, Qu XD, Chen XJ, Lu HS, Wang Y, et al. Aloesin suppresses cell growth and metastasis in ovarian cancer SKOV3 cells through the inhibition of the MAPK signaling pathway. Anal Cell Pathol (Amst) 2017;2017:8158254

14. Ahirwar K, Jain SK. Aloe-emodin novel anticancer herbal drug. Int J Phytomed 2011;3:27-31.

15. Kumar NA, Sharmila R, Akila K, Jaikumar B. In-silico approach for the assessment of oral cancer property on Limonia acidissima. Int J Pharm Sci Res 2016;7:1271-75.

16. Sanghani HV, Ganatra SH, Pande R. Molecular-docking studies of potent anticancer agent. J Comput Sci Syst Biol 2012;5:12-5.

17. Norleena PG, Amin AR, Bayraktar S, Pezzuto JM, Shin DM, Khuri FR, et al. Cancer prevention with natural compounds. Semin Oncol 2010;37:258-81.

18. Huang Q, Lu G, Shen HM, Chung MC, Ong CN. Anti-cancer properties of anthraquinones from rhubarb. Med Res Rev 2007;27:609-30.

19. Adhikari A, Mahar KS. DNA targeted anthraquinone derivatives: An important anticancer agent. Int J Pharm Pharm Sci 2016;8:17-25.

20. Bairam R, Muppavarapu SM, Sreekanth S. Synthesis, characterization, biological evaluation and docking of some novel substituted 1, 3-thiazine derivatives. Int J Pharm Pharm Sci 2017;9:233-42. 